\title{
Non-Invasive Brain Stimulation in Children With Unilateral Cerebral Palsy: A Protocol and Risk Mitigation Guide
}

\author{
Bernadette T. Gillick ${ }^{1 *}$, Andrew M. Gordon ${ }^{2}$, Tim Feyma ${ }^{3}$, Linda E. Krach ${ }^{4}$, Jason Carmel', \\ Tonya L. Rich ${ }^{6}$, Yannick Bleyenheuft ${ }^{7}$ and Kathleen Frie/ ${ }^{5}$ \\ 'Physical Therapy Division, Department of Rehabilitation Medicine, University of Minnesota, Minneapolis, MN, United States, \\ ${ }^{2}$ Department of Biobehavioral Sciences, Teachers College, Columbia University, New York, NY, United States, ${ }^{3}$ Gillette \\ Children's Specialty Healthcare, Pediatric Neurology, St. Paul, MN, United States, ${ }^{4}$ Courage Kenny Rehabilitation Institute, \\ Minneapolis, MN, United States, ${ }^{5}$ Weill-Cornell Medical College, Blythedale Children's Hospital, Burke Medical Research \\ Institute, White Plains, NY, United States, ${ }^{6}$ Rehabilitation Science, University of Minnesota, Minneapolis, MN, United States, \\ ${ }^{7}$ Institute of Neuroscience (IoNS), Universite catholique de Louvain, Brussels, Belgium
}

OPEN ACCESS

Edited by:

Carl E. Stafstrom, Johns Hopkins Medicine,

United States

Reviewed by:

Gouri Rao Passi,

Choithram Hospital and

Research Centre, India

Ryan J. Felling,

Johns Hopkins University,

United States

*Correspondence:

Bernadette T. Gillick

gillick@umn.edu

Specialty section:

This article was submitted to

Pediatric Neurology,

a section of the journal

Frontiers in Pediatrics

Received: 25 August 2017 Accepted: 26 February 2018

Published: 16 March 2018

Citation:

Gillick BT, Gordon AM, Feyma T,

Krach LE, Carmel J, Rich TL, Bleyenheuft $Y$ and Friel K (2018) Non-Invasive Brain Stimulation in Children With Unilateral Cerebral

Palsy: A Protocol and Risk Mitigation Guide.

Front. Pediatr. 6:56.

doi: 10.3389/fped.2018.00056
Non-invasive brain stimulation has been increasingly investigated, mainly in adults, with the aims of influencing motor recovery after stroke. However, a consensus on safety and optimal study design has not been established in pediatrics. The low incidence of reported major adverse events in adults with and without clinical conditions has expedited the exploration of NIBS in children with paralleled purposes to influence motor skill development after neurological injury. Considering developmental variability in children, with or without a neurologic diagnosis, adult dosing and protocols may not be appropriate. The purpose of this paper is to present recommendations and tools for the prevention and mitigation of adverse events (AEs) during NIBS in children with unilateral cerebral palsy (UCP). Our recommendations provide a framework for pediatric NIBS study design. The key components of this report on NIBS AEs are (a) a summary of related literature to provide the background evidence and (b) tools for anticipating and managing AEs from four international pediatric laboratories. These recommendations provide a preliminary guide for the assessment of safety and risk mitigation of NIBS in children with UCP. Consistent reporting of safety, feasibility, and tolerability will refine NIBS practice guidelines contributing to future clinical translations of NIBS.

\footnotetext{
Keywords: cerebral palsy, non-invasive brain stimulation, transcranial magnetic stimulation, transcranial direct current stimulation, repetitive transcranial magnetic stimulation, safety, risk, children
}

\section{BACKGROUND}

Neuromodulatory interventions using non-invasive brain stimulation (NIBS) have been increasingly investigated aiming to influence cortical excitability non-surgically in myriad pediatric neurologic conditions including stroke, epilepsy, and unilateral cerebral palsy (UCP) (1). Two forms of NIBS include: (1) electromagnetic induction using transcranial magnetic stimulation (TMS) as an assessment or test of cortical excitability or, when applied repetitively, repetitive transcranial magnetic stimulation (rTMS) as an intervention and (2) electrical current using transcranial direct current stimulation (tDCS) as an intervention. In typically developing children, NIBS has also been 
investigated as a means to enhance neural plasticity and improve learning $(2,3)$. As an adjuvant technology, NIBS holds potential to enhance existing pediatric rehabilitation interventions more rapidly and cost-effectively than current practice. However, a consensus surrounding safety, applications, and ethics in the incorporation of NIBS in these conditions has not been established, and guidelines for optimal study design construct are limited. Across four international laboratories, incorporating studies of over 225 children and hundreds of NIBS sessions, we share and integrate current and related pediatric NIBS literature with our protocols in UCP as a means to consider individual variation to mitigate risk and manage adverse events (AEs) in this specific population.

Transcranial magnetic stimulation and rTMS utilize the principles of electromagnetic induction through the use of a coil on the participant's head over the underlying region of interest. For example, stimulating by placing the TMS coil overlying the area of the primary motor cortex may elicit a motor-evoked potential response in a corresponding hand muscle. If the intent is to test or assess cortical excitability, TMS can be employed in protocols incorporating single or paired pulses (4). This allows for non-surgical assessment of current neurophysiologic status and can be integrated into assessments surrounding neuromodulatory and behavioral interventions. If the intent is to neuromodulate through an intervention, rTMS provides repeated TMS pulses with the aim of inducing a change in cortical excitability: facilitation or inhibition. rTMS, from a neurophysiologic perspective, therefore acts as an intervention to induce action potentials to influence cortical excitability.

Transcranial direct current stimulation applies weak electrical pulses through surface scalp electrodes, at least one anode and cathode, with different montages and dosages (5). Although not strong enough to produce a motor-evoked potential, the current can alter the resting membrane potential, influencing the excitability in endogenous firing rate (6). tDCS may therefore bias cortical activation toward depolarization or hyperpolarization and act as a catalyst to prime the brain for activation and rehabilitation.

Within the context of the developing brain with neurologic injury and underlying neurophysiology, NIBS has been found to influence cortical and behavioral responses. In the case of a unilateral brain injury (e.g., perinatal stroke with resultant UCP) wherein an imbalance between the developing hemispheres may occur, such neuromodulatory interventions may provide a stimulus for integration of dormant yet viable penumbral-region neuronal activity. Added contributions from these ipsilesional neurons may, in turn, allow further development of motor function. In UCP, the application can then either facilitate excitability of one hemisphere (e.g., the lesioned hemisphere) or reduce exaggerated inhibitory effects from an area such as the non-lesioned hemisphere.

In other disorders of cortical excitability, e.g., epilepsy, the target may indeed be the region of the seizure with a stimulation protocol intended to inhibit seizure activity.

Dosing for the forms of interventional NIBS aforementioned varies based on the aims of the protocol and the intent to inhibit or excite by neuromodulation. Dosing has been described in detail in the pediatric population (7). In brief, investigating rTMS in pediatric populations, intensity ranges have been reported to vary between sham settings $(0 \mathrm{~Hz})$ and $6 \mathrm{~Hz}$. For tDCS, intensity ranges from sham $(0.0 \mathrm{~mA})$ to $2.0 \mathrm{~mA}$ settings. For both rTMS and tDCS, the duration of a single session has been reported up to $20 \mathrm{~min}$, with a maximum of 10 daily serial sessions.

Recent computational modeling, investigating dosing and the cortical field induced by NIBS, has guided research protocols with the goal of assessing the safety, feasibility, and efficacy of such interventions in adults and children $(8,9)$. NIBS can be paired with adjuvant interventions such as upper- and lowerextremity intensive therapies, developing a synergistic application which may advance improvements in the function of the more affected extremities (10). As NIBS mechanisms differ, allowing targeting for specific applications such as priming the nervous system for optimization of therapy (11) or enhancing motor learning (12).

As a means to further explore individual pediatric participant considerations to optimize study design, we investigated the reporting of NIBS-related serious adverse events (SAEs) and minor adverse events (MAEs) in the current pediatric literature, specifically in children with cerebral palsy (CP) inclusive of all subtypes. Table 1 provides a summary of pediatric neuromodulation studies and the reporting of adverse events.

\section{Existing Safety Guidelines and Protocols}

Although summaries and systematic reviews of NIBS studies in both adult and child populations have been published, a consensus as to safe NIBS application in children with UCP, considering brain reorganization, development, and the potential for resultant seizure, has not yet been established $(1,4,5,68-73)$. While the NIBS protocols we have generated feature diagnosis and lesion-specific study designs for children with UCP, we suggest that these recommendations may be applicable as well to NIBS protocols in children with other clinical presentations of $\mathrm{CP}$, such as tetraparesis or diparesis. The indications for such a guide arise from questions as to how NIBS might be applied differently to individuals with UCP based on their lesions or spared neuronal circuitry, as well as from the issue of dosing based on head size and effective current. Additionally, this guide, created from our combined pediatric research, may help to establish optimal practice guidelines and future comprehensive protocols for studies incorporating participants with congenital UCP and postnatally acquired conditions with a clinical presentation of UCP.

Serious adverse events reported as related to NIBS are mainly seizure and syncope. TMS-related seizures have been reported directly during or after intervention in children with depression, however, specific to the small samples in pediatric UCP, there have been no reported seizures with either TMS testing or rTMS interventions $(1,70)$. From the few reports of adults experiencing seizure during single-pulse TMS session applications, many had a pre-existing brain lesion and a diagnosis of intractable epilepsy (68).

Distinct from seizures induced by other forms of NIBS, such as direct electrical cortical stimulation (70), tDCS applications are often employed with the intent to improve seizure control in both children and adults (74), and typically no SAEs (seizure or syncope) have been reported with tDCS interventions (5). However, 
TABLE 1 | Summary of pediatric neuromodulation studies and reported adverse events.

\begin{tabular}{|c|c|c|c|}
\hline Reference & $\begin{array}{c}\text { AE } \\
\text { addressed }\end{array}$ & Sample size & Withdraws \\
\hline \multicolumn{4}{|l|}{ TMS } \\
\hline Brouwer and Ashby (13) & No & $\begin{array}{l}4 \text { children (10 total } \\
\text { with } \mathrm{CP}, 22 \\
\text { additional controls) }\end{array}$ & 0 \\
\hline Farmer et al. (14) & No & 2 (of 4 children) & 0 \\
\hline Carr et al. (15) & No & $\begin{array}{c}33 \text { (ages 2-26, not } \\
\text { defined) }\end{array}$ & 0 \\
\hline Maegaki et al. (16) & Yes & $\begin{array}{c}8 \text { children } \\
\text { (12 additional either } \\
\text { adults or later-onset } \\
\text { lesions) }\end{array}$ & 0 \\
\hline Heinen et al. (17) & No & $\begin{array}{c}6 \text { children (4 other } \\
\text { controls) }\end{array}$ & 0 \\
\hline Maegaki et al. (18) & Yes & 17 & 0 \\
\hline Nezu et al. (19) & No & 9 & 0 \\
\hline Yasuhara et al. (20) & Yes & 9 & 0 \\
\hline Thickbroom et al. (21) & No & 7 & 0 \\
\hline Staudt et al. (22) & No & 12 & 2 \\
\hline Eyre et al. (23) & No & 39 & 0 \\
\hline Berweck et al. (24) & Yes & $\begin{array}{c}7 \text { children (10 total } \\
\text { with congenital } \\
\text { hemiparesis, } 8 \\
\text { additional controls) }\end{array}$ & 0 \\
\hline Kuhnke et al. (25) & Yes & 9 & 0 \\
\hline Redman et al. (26) & Yes & 22 & 2 \\
\hline Staudt et al. (27) & No & 1 child (8 total) & 0 \\
\hline Vry et al. (28) & Yes & 15 & 1 \\
\hline Pilato et al. (29) & No & 1 & 0 \\
\hline Walther et al. (30) & No & 7 & 0 \\
\hline Wilke et al. (31) & No & 14 & 0 \\
\hline Wittenberg (32) & No & 10 & 0 \\
\hline Holmström et al. (33) & Yes & 17 & 0 \\
\hline Kesar et al. (34) & No & 13 & 0 \\
\hline van der Aa et al. (35) & No & 37 & 0 \\
\hline Yang et al. (36) & Yes & 5 & 1 \\
\hline Flamand and Schenider (37) & No & 1 & 0 \\
\hline Islam et al. (38) & No & 13 of 16 & 3 \\
\hline Mackey et al. (39) & No & 12 & 0 \\
\hline Pinko et al. (40) & No & $\begin{array}{l}10 \text { children (of a total } \\
\text { of } 12 \text { children with } \\
\text { CP and } 12 \text { TDC) }\end{array}$ & Not stated \\
\hline Bleyenheuft et al. $(10,41)$ & Yes & 2 & 0 \\
\hline Narayana et al. (42) & Yes & 2 & 0 \\
\hline Baranello et al. (43) & No & 17 & 0 \\
\hline Friel et al. (44) & Yes & 20 & 0 \\
\hline Kuo et al. (45) & No & 20 & 0 \\
\hline
\end{tabular}

\section{rTMS}

Valle et al. (46)

Kirton et al. $(47,48)$

Gillick et al. (8, 49)

Kirton et al. (50)

Guo et al. (51)

Gupta et al. (52)

\section{tDCs}

Young et al. (53)

Aree-uea et al. (54)

Duarte et al. (55)

Ferreira et al. (56)

Grecco et al. (57-59)

$\begin{array}{lcl}\text { Yes } & 17 & 0 \\ \text { Yes } & 10 & 0 \\ \text { Yes } & 19 & 0 \\ \text { Yes } & 45 & 0 \\ \text { No } & 1 & 0 \\ \text { Yes } & 20 & 0\end{array}$

Yes

\section{Yes}

Yes

Yes

No

Yes

$\begin{array}{ll}11 & 1 \\ 46 & 1 \\ 24 & 0 \\ 12 & 0 \\ 24 & 0\end{array}$

(Continued)
TABLE 1 | Continued

\begin{tabular}{lccc}
\hline Reference & $\begin{array}{c}\text { AE } \\
\text { addressed }\end{array}$ & Sample size & Withdraws \\
\hline Grecco et al. (57-59) & Yes & 20 & 0 \\
Grecco et al. (57-59) & Yes & 1 & 0 \\
Young et al. (60) & Yes & 14 & 0 \\
Bhanpuri et al. (61) & Yes & 6 (of 9 total) & 0 \\
Ekici (62) & Yes & 1 & 1 \\
Gillick et al. (63) & Yes & 13 & 1 \\
Grecco et al. (64) & Yes & 20 & 1 \\
Lazzari et al. (65) & No & 20 & 0 \\
Carvalho Lima et al. (66) & Yes & 1 & 0 \\
Grecco et al. (67) & Yes & 6 & 0
\end{tabular}

$A E$, adverse events; $C P$, cerebral palsy; N/A, not applicable; rTMS, repetitive transcranial magnetic stimulation; tDCS, transcranial direct current stimulation; TDC, typically developing children; TMS, transcranial magnetic stimulation.

a recent report revealed a seizure after a third consecutive session in a daily tDCS protocol in a 4-year-old child with spastic tetraparesis (62). The child, who had a history of seizures and was in the process of adjusting valproate and tapering of topiramate, had been seizure-free for the previous year and reportedly remained seizure-free after the cessation of the tDCS and involvement in the protocol. The direct correlation between tDCS and the incidence of a seizure in this single-case example is difficult to determine.

Two neurocardiogenic syncopal episodes, during initial TMS testing sessions, have been reported in children with UCP (47). In both cases, a history of fainting or previous syncope was reported after the event.

\section{SAEs Mitigation Recommendations}

Seizure management is complex where consensus on one unified approach for selecting and identifying a plan for rescue medications does not exist. Administration of rescue medications requires medically trained caregivers and/or investigators. The selection of rescue medications is typically guided by a pediatric neurologist based on the duration, type, and history of seizures. Several options exist for emergency rescue medication protocols. One option may be emergency administration of rescue medications (e.g., rectal Diastat with a verified appropriate dose based on the child's weight). Another option is for an alternate rescue medication, such as intranasal Midazolam, which is less intrusive than rectal Diastat (Diazepam) and potentially indicated for children with seizures of a very short duration. A third option is to utilize emergency medical services, allowing for assessment in an emergency room with a physician on staff. Site-specific selection of the seizure action plan depends on (1) recommendations from medical monitor, medical director, and ongoing treating neurologist, (2) training of investigators, and (3) institutional review board (IRB) requirements.

Both seizure management plans and observation records should be established (Appendices A and B in Supplementary Material). If a seizure does occur during study participation, we recommend added documentation for the family and child to represent the isolated seizure as most likely a study-related event. For example, a letter from the study physician (medical director) explaining the NIBS intervention and clarifying the details 
of the seizure event, may guide follow-up medical management (Appendix C in Supplementary Material).

To minimize risk of syncope, Kirton et al. specifically recommended the following: (1) screening for previous episodes of syncope, fainting history, and evaluation of why the episodes occurred, (2) adequate food/water intake prior to participation in an NIBS study, and (3) monitoring during the study for dizziness and nausea (47).

Minor adverse events commonly reported in adults and children during NIBS include headache, dizziness, neck pain, and scalp irritation (1).

\section{Testing: TMS MAEs}

To examine MAE in studies involving children, a literature search yielded 33 studies incorporating TMS in 401 children with CP (13-21, 23-25, 27-36, 38-40, 42-45, 75). Twelve of those studies (36\%) addressed or mentioned AEs (Table 1). Four studies (12\%) reported a total of nine MAEs, which included discomfort, headache, and decreased tolerance-related directly to the TMS testing (Table 1).

\section{Intervention: rTMS MAEs}

Six rTMS studies have been published using rTMS as an intervention in 112 total children with CP $(46,48,49,50-52)$. Of those six studies, five addressed or mentioned AEs (83\%) (Table 1). Four studies (67\%) formally reported a total of 57 complaints of MAEs, which included worsening of sleep, social function, mobility, vegetative symptoms, headache, anxiety, dizziness, tingling, mood changes, concentration changes, abnormal muscle contractions, nausea, stomachache, fatigue, and tingling (Table 1). There is wide variability in the reporting of AEs in both real and control groups. It is important to note the percentages of children in the control groups and including assessment of their tolerance of the intervention is central to enrollment and retention of pediatric participants.

\section{Intervention: tDCS MAEs}

Specific to tDCS in children with CP, MAEs commonly include headache, dizziness, and scalp-related complaints (e.g., burning, abrasion, tingling, and itching) (1). Fifteen studies, of a total of 219 children with CP, incorporated tDCS $(63,53-62,64-67)$. Thirteen (87\%) addressed or mentioned AEs (Table 1). Eleven (73\%) reported a total of 48 MAEs, which included complaints of discomfort, rash, burn, itching, sleepiness, tingling, redness, and pain (Table $\mathbf{1}$ ).

\section{MAEs Mitigation}

Risk mitigation plans are essential for use by the investigators for preparation and response if an $\mathrm{AE}$, on any level, does occur (Appendices D-F in Supplementary Material). Proper screening and assessment of the potential for MAEs are imperative as these events give clues as to the tolerance a child may have for NIBS. Mitigation begins at enrollment; specific pediatric-based considerations regarding the developmental and clinical status of the child involved in an NIBS protocol include the size and location of the lesion or region of interest, the natural history of the disorder, selection of assessment tools [such as neurologist assessment, e.g., modified Pediatric Stroke Outcome Measure (76)], and an outcome tool [motor function/activity measures, e.g., Assisting Hand Assessment (77)]. Age appropriate questionnaires assessing symptoms can be completed in 5-10 min with children and caregivers. Additionally, we recommend offering breaks/snacks/ non-caffeinated beverages, and assessment of nonverbal cues of discomfort (e.g., wincing). Incorporating routine stops during NIBS allows further assessment to further establish if the child feels nauseated, dizzy, or uncomfortable. Careful assessment of AEs, both serious and minor, allows for appropriate management and also for evaluation in the design of potential future interventions.

\section{AEs Reporting Recommendations}

The potential for AEs should be conveyed not only in writing, both on the consent (caregiver) and assent (child) forms, but also verbally. Age-specific and cognitive level appropriate language should be used in AE discussion and interpretation of the scientific literature surrounding NIBS interventions. Integrating feedback from well-informed caregivers before, during, and after the study can be essential to ensure accuracy and completeness of reporting. Inclusion of a quality-of-life measure, depression inventory, or a neuropsychological assessment tool, e.g., Weinberg Depression Scale (78), may be indicated to establish a baseline understanding of the child's status and potential changes when working with NIBS.

A lack of reporting AEs related to NIBS studies is not necessarily a statement that they did not occur, rather that they may not have been addressed or reported. The comprehensive reporting of such details surrounding an intervention allows not only for potential replication or building upon study findings but also for the opportunity to safely and reliably implement interventions with the intent to benefit patient outcomes (79).

\section{STUDY DESIGN CONSIDERATIONS}

An understanding of NIBS safety and limitations guides the researcher when constructing optimal pediatric study designs and developing protocols for such analyses. However, studiesto-date vary, without consensus, in establishing participation criteria, monitoring methods, and providing optimal environments. As research progresses toward consensus, we recommend consideration of, at minimum, the following components: criteria for participant safety, safety review committees, control groups, environmental acclimation, and follow-up assessments.

\section{Study Criteria for Participant Safety}

We propose practical consideration, from the outset, of the general study criteria when developing the study design. First, an understanding of the safety contraindications for NIBS is imperative for appropriate participant selection. Contraindications start with identifying indwelling metal and/or medical devices. Case by case review of implanted ferromagnetic and/or medical devices is indicated. We therefore currently devise our safety protocols based upon adult NIBS outcomes and upon an understanding of the effect of NIBS on the underlying cortex. We propose a thorough screening of the child's medical history, with specific emphasis on diagnosis, history of seizure, syncope, headache, as 
well as cognitive deficits and behavioral disorders, prior to inclusion in any NIBS study. We have found variability in working with our respective IRBs in terms of study criteria boundaries, but fundamental safety concerns apply when considering candidates for NIBS, whether TMS, rTMS, or tDCS. Table 2 displays the study criteria similarities among our IRB-approved laboratory protocols (Table 2 study criteria).

Many of the current guidelines for NIBS in children are derived from the adult stroke literature. However, complete adoption of adult protocols may be inappropriate (e.g., inclusion/exclusion criterion). For example, specific to epilepsy screening and variation between ages, the incidence of epilepsy in adults with stroke is $2.5 \%$ (80) whereas in children with UCP, the incidence of epilepsy has been reported at $26 \%$ (81). Additionally, based on available data, a child's risk of seizure recurrence with focal epilepsy has largely stabilized at 2 years of age (82).

Inclusion guidelines for pediatric NIBS participants with a history of epilepsy are not well established in the literature nor does a uniform criterion between pediatric studies exist. The strength of the limited, yet available evidence in establishing guidelines is variable. However, the safety profile for pediatric TMS has recently been reported to be a risk of seizure $0.14 \%$ per session (4). Policies established at individual institutions and regulatory oversight mechanisms may reflect different protocols. Indeed, between our own laboratories at different sites, we have discovered variations in the existing study criteria $(49,63,83)$. One approach is to include children of any age who have been seizure-free for 2 years. This is based on clinical discussion of medication withdrawal, as well as mechanism of injury, and ongoing EEG positivity $(82,84)$. Based on the risk-recurrence data available for the child with epilepsy, another approach is to exclude children with any history of seizures after 2 years of age

TABLE 2 | Study criteria for NIBS study eligibility for children with CP.

\begin{tabular}{ll}
\hline Inclusion criteria & Exclusion criteria \\
\hline - Congenital unilateral cerebral palsy & - Metabolic disorders \\
secondary to periventricular leukomalacia & - Neoplasm \\
or perinatal stroke confirmed by most & - Acquired traumatic brain injury \\
recent MRI or CT radiologic report with & - Pregnancy \\
resultant congenital hemiparesis & - Indwelling metal or incompatible \\
- Receptive language function to follow & medical devices \\
two-step commands & - Evidence of skin disease or skin \\
- A determination by the investigators & abnormalities \\
and institutions, as to the time since last & - Spasticity injections such as \\
seizure or age since seizure-free, with & Botulinum Toxin or Phenol Block \\
documentation of all current medications & within the previous 6 months so \\
to include anti-epileptic medications & as not to potentially influence \\
- Presence of a motor-evoked potential & outcomes related to NIBS \\
from at least the contralesional hemisphere & intervention \\
(if not both hemispheres) for measurement & \\
of baseline cortical excitability and as a & \\
guide for site of stimulation & \\
- Able to give informed assent along with & \\
the informed consent of a legal guardian & \\
- Children who have surgeries, which may & \\
influence motor function (e.g., tendon & \\
transfers). Surgical history should be & \\
documented & \\
\hline
\end{tabular}

MRI, magnetic resonance imaging, CT, computerized tomography.
$(41,44,85)$. This approach includes children who may have only presented with a seizure at the time of brain insult, but who have had no further seizures beyond age 2 years. The approach is also based on the fact that seizure incidence is highest in the first year of life and many children will only have one seizure which occurs at the time of birth (86).

\section{Safety Review Committee}

A designated medical monitor who is not an investigator on the study but who has experience in review of symptoms (e.g., neurologist, pediatric rehabilitation medicine physician) may be included. This monitor could review child status before the study, at a defined interim and after the study is completed. Additionally, the medical monitor can be contacted to review case by case when an AE occurs. A Data Safety Monitoring Board (DSMB) could also be employed with a group of non-studyrelated professionals whose members (i.e., physicians, statisticians, academicians, and pediatric researchers) are familiar with NIBS and potential AEs, the study design, or congenital UCP. This board can provide interim feedback as to study continuation or cessation and review participant status. Presentation to the DSMB can occur in a blinded manner, with all investigators present, and in an additional unblinded session wherein the unblinded investigators can discuss with the board potential differences between individuals and groups.

\section{Control Group}

The inclusion of a control group of children with and without UCP can allow for group-based comparisons. IRB approval for including children with typical development may depend on the potential to demonstrate direct benefit. Developing an institutional summary of the evidence of NIBS trials in children with typical development displaying the low rate of AEs may aid in gaining IRB approval. Within-participant comparison could occur with crossover designs but may be prohibitive from a feasibility and time-commitment standpoint for families. It has been found, in both adults and children, that participants are unaware of which form of stimulation was received $(49,87)$. Additionally, if a child with a unilateral brain lesion (e.g., perinatal stroke) is involved in an NIBS intervention targeting one hemisphere or both, the non-lesioned hemisphere and less-affected extremities should be monitored either as a control or a comparison for potential changes in function and safety.

\section{Acclimation to the NIBS Environment}

Through focus polls and feedback from families and children with UCP, our labs have learned that interactions, from the moment the family becomes aware of the study/research, have a strong influence on participation or non-participation decisions. Children and their caregivers need the opportunity to understand the study, directions, and the demands of the protocol. To allow families to acclimate to study-related information, eligibility surveys and follow-up discussions may be conducted over the phone. Allocation of adequate time for consent and assent procedures builds in time for questions and establishes an environment that allows for additional discussion and questions. Children and families considering participation can be connected with past 
participants of an NIBS study, pending agreement from both families. Additionally, the study environment can be adapted, by pediatric appropriate seating and decoration, to be esthetically pleasing and less anxiety-producing to the child, whenever this opportunity is available.

Pediatric size earplugs provide protection for hearing from the frequency of the TMS (68). Hearing loss was reported in one participant when using an H-coil (88). Due to paucity of research investigating the impact of TMS on pediatric auditory function, we continue to employ and recommend appropriate hearing protection based on the recommendations from Rossi et al. (68), given the developing auditory system as well as the smaller size of the pediatric head resulting in the coil being closer to the ear.

In order for a child to acclimate to tDCS, ramp-up and rampdown features, as well as a pre-stimulation pulse, can be used. If stimulation thresholds or excitability data are being used as an outcome, ensure that there are no systematic differences in pretest and posttest environments.

\section{Follow-Up Assessment}

Although a child may not have reported or experienced AEs throughout the study, additional feedback from the child/ caregiver can improve future study design. A satisfaction survey, such as that adapted from Garvey et al. $(89,90)$, can assist in analysis of participant satisfaction in the study. A confidential family feedback form, filled out anonymously by the child and caregiver together, can provide helpful information (Appendix $\mathrm{G}$ in Supplementary Material). Additionally, formal follow-up at established time points after the study allows long-term assessment of participant status and interpretation of the potential longitudinal effects that NIBS may have (91).

\section{CONCLUSION}

As evident from the above discussion and AEs, the study design and protocol are crucial elements in guiding NIBS assessments and interventions. Adaptation of supporting equipment and environment may improve the comfort of the child. A thorough medical history, as well as assessment of the caregiver's and child's understanding of the protocol, can guide discussions and interpretation of the long-term impact of these interventions. Finally, allowing ample time and opportunity for a child to experience NIBS and give feedback is integral to successful participant enrollment and retention.

NIBS and applications in both typically developing children and those with neurologic diagnoses provide a unique means to establish a "window into the brain," assessing cortical excitability, mapping, and reorganization. Interventions which incorporate energy transfer to the developing brain, with or without neurologic lesion, must exercise extreme caution not only for the individuals involved but also for advancement of the field. Further considerations include recognition of the anatomic and physiologic differences between an adult brain and a child brain underscoring the need for rigorous investigation before implementation. We present here a comprehensive account of what we have learned to date through previous published research and through collaboration in the work of our laboratories and we offer recommendations for uniformity in reporting of research studies. Key elements include a precise understanding of the organization of the developing brain with a congenital lesion, integration of optimal indications for study participation, incorporation of AEs profile and mitigation, as well as multi-dimensional, longitudinal, study design. Combining our experiences has allowed us to improve our own protocols, with the immediate goal of child safety and the overarching goal to establish a consensus that helps to define best NIBS practice and practice guidelines. Examining both the safety and the feasibility of NIBS studies offers the optimal manner in which to investigate the effectiveness of our interventions.

\section{ETHICS STATEMENT}

This study was carried out in accordance with the recommendations of Institutional Review Board with written informed consent from all participants. All participants gave written informed consent in accordance with the Declaration of Helsinki. The protocol was approved by the Institutional Review Board.

\section{AUTHOR CONTRIBUTIONS}

BG, AG, TF, LK, JC, TR, YB, and KF contributed to the design of this work, writing and revisions of this work, final approval, and agreed to be accountable for all aspects of the work.

\section{ACKNOWLEDGMENTS}

Importantly, we are grateful to the caregivers and children in our studies who made this work, and understanding, possible. We kindly acknowledge the editing and proofing of Sally Jones.

\section{FUNDING}

Funding sources are 1UL1RR033183-01NCRR (BG), 8UL1TR000114-02 (BG), UL1TR000114 NCATS (BG), NIH NICHD 1K01HD078484-01A1 (BG), MN Med Fnd (BG), FPT (BG), CPF (BG), NS062116 (KF), Columbia Professional Schools Diversity Award (KF), NIH CTSA Award (KL2 RR024157, UL1 RR024156, TL1 RR024158) (KF), HD076436 (KF), NIH NINDS K08 NS073796 (JC), AACPDM Student Travel award (TR), and UMN MnDRIVE Fellowship (TR).

\section{SUPPLEMENTARY MATERIAL}

The Supplementary Material for this article can be found online at http://www.frontiersin.org/articles/10.3389/fped.2018.00056/ full\#supplementary-material.

APPENDIX A | Seizure Management.

APPENDIX B | Seizure Observation Record.

APPENDIX C | Seizure Event Letter.

APPENDIX D | Transcranial Direct Current Stimulation (tDCS) Risk Mitigation.

APPENDIX E | Transcranial Magnetic Stimulation (TMS) Risk Mitigation.

APPENDIX F | Participant Report of Symptoms.

APPENDIX G | Confidential family feedback form. 


\section{REFERENCES}

1. Krishnan C, Santos L, Peterson MD, Ehinger M. Safety of noninvasive brain stimulation in children and adolescents. Brain Stimul (2015) 8(1):76-87. doi:10.1016/j.brs.2014.10.012

2. Maslen H, Earp BD, Cohen Kadosh R, Savulescu J. Brain stimulation for treatment and enhancement in children: an ethical analysis. Front Hum Neurosci (2014) 8:1-5. doi:10.3389/fnhum.2014.00953

3. Ciechanski P, Kirton A. Transcranial direct-current stimulation can enhance motor learning in children. Cereb Cortex (2017) 27(5):2758-67. doi:10.1093/ cercor/bhw114

4. Allen CH, Kluger BM, Buard I. Safety of transcranial magnetic stimulation in children: a systematic review of the literature. Pediatr Neurol (2017) 68:3-17. doi:10.1016/j.pediatrneurol.2016.12.009

5. Bikson M, Grossman P, Thomas C, Zannou AL, Jiang J, Adnan T, et al. Safety of transcranial direct current stimulation: evidence based update 2016. Brain Stimul (2016) 9(5):641-61. doi:10.1016/j.brs.2016.06.004

6. Nitsche MA, Cohen LG, Wassermann EM, Priori A, Lang N, Antal A, et al. Transcranial direct current stimulation: state of the art 2008. Brain Stimul (2008) 1(3):206-23. doi:10.1016/j.brs.2008.06.004

7. Gillick B, Friel K, Menk J, Rudser K. Therapeutic brain stimulation trials in children with cerebral palsy. In: Kirton A, Gilberg D, editors. Pediatric Brain Stimulation Mapping and Modulating the Developing Brain. Amsterdam: Elsevier (2016). p. 210-34.

8. Gillick BT, Kirton A, Carmel JB, Minhas P, Bikson M. Pediatric stroke and transcranial direct current stimulation: methods for rational individualized dose optimization. Front Hum Neurosci (2014) 8:739. doi:10.3389/fnhum. 2014.00739

9. Bikson M, Rahman A, Datta A. Computational models of transcranial direct current stimulation. Clin EEG Neurosci (2012) 43(3):176-83. doi:10.1177/ 1550059412445138

10. Bleyenheuft Y, Arnould C, Brandao MB, Bleyenheuft C, Gordon AM. Hand and arm bimanual intensive therapy including lower extremity (HABIT-ILE) in children with unilateral spastic cerebral palsy. Neurorehabil Neural Repair (2015) 29(7):645-57. doi:10.1177/1545968314562109

11. Carey JR, Deng H, Gillick BT, Cassidy JM, Anderson DC, Zhang L, et al. Serial treatments of primed low-frequency rTMS in stroke: characteristics of responders vs. nonresponders. Restor Neurol Neurosci (2014) 32(2):323-35. doi:10.3233/RNN-130358

12. Reis J, Schambra HM, Cohen LG, Buch ER, Fritsch B, Zarahn E, et al. Noninvasive cortical stimulation enhances motor skill acquisition over multiple days through an effect on consolidation. Proc Natl Acad Sci U S A (2009) 106(5):1590-5. doi:10.1073/pnas.0805413106

13. Brouwer B, Ashby P. Altered corticospinal projections to lower limb motoneurons in subjects with cerebral palsy. Brain (1991) 114(Pt 3):1395-407. doi:10.1093/brain/114.3.1395

14. Farmer SF, Harrison LM, Ingram DA, Stephens JA. Plasticity of central motor pathways in children with hemiplegic cerebral palsy. Neurology (1991) 41(9):1505-10. doi:10.1212/WNL.41.9.1505

15. Carr LJ, Harrison LM, Evans AL, Stephens JA. Patterns of central motor reorganization in hemiplegic cerebral palsy. Brain (1993) 116(Pt 5):1223-47. doi:10.1093/brain/116.5.1223

16. Maegaki Y, Maeoka Y, Ishii S, Shiota M, Takeuchi A, Yoshino K, et al. Mechanisms of central motor reorganization in pediatric hemiplegic patients. Neuropediatrics (1997) 28(3):168-74. doi:10.1055/s-2007-973695

17. Heinen F, Glocker FX, Fietzek U, Meyer BU, Lücking CH, Korinthenberg R. Absence of transcallosal inhibition following focal magnetic stimulation in preschool children. Ann Neurol (1998) 43(5):608-12. doi:10.1002/ana. 410430508

18. Maegaki Y, Maeoka Y, Ishii S, Eda I, Ohtagaki A, Kitahara T, et al. Central motor reorganization in cerebral palsy patients with bilateral cerebral lesions. Pediatr Res (1999) 45(4 Pt 1):559-67. doi:10.1203/00006450-199904010-00016

19. Nezu A, Kimura S, Takeshita S, Tanaka M. Functional recovery in hemiplegic cerebral palsy: ipsilateral electromyographic responses to focal transcranial magnetic stimulation. Brain Dev (1999) 21(3):162-5. doi:10.1016/ S0387-7604(98)00094-1

20. Yasuhara A, Niki T, Ochi A. Changes in EEG after transcranial magnetic stimulation in children with cerebral palsy. Electroencephalogr Clin Neurophysiol Suppl (1999) 49:233-8.
21. Thickbroom GW, Byrnes ML, Archer SA, Nagarajan L, Mastaglia FL. Differences in sensory and motor cortical organization following brain injury early in life. Ann Neurol (2001) 49(3):320-7. doi:10.1002/ana.68

22. Staudt M, Grodd W, Gerloff C, Erb M, Stitz J, Krägeloh-Mann I. Two types of ipsilateral reorganization in congenital hemiparesis: A TMS and fMRI study. Brain (2002) 125(10):2222-37. doi:10.1093/brain/awf227

23. Eyre JA, Smith M, Dabydeen L, Clowry GJ, Petacchi E, Battini R, et al. Is hemiplegic cerebral palsy equivalent to amblyopia of the corticospinal system? Ann Neurol (2007) 62(5):493-503. doi:10.1002/ana.21108

24. Berweck S, Walther M, Brodbeck V, Wagner N, Koerte I, Henschel V, et al. Abnormal motor cortex excitability in congenital stroke. Pediatr Res (2008) 63(1):84-8. doi:10.1203/PDR.0b013e31815b88f1

25. Kuhnke N, Juenger H, Walther M, Berweck S, Mall V, Staudt M. Do patients with congenital hemiparesis and ipsilateral corticospinal projections respond differently to constraint-induced movement therapy? Dev Med Child Neurol (2008) 50(12):898-903. doi:10.1111/j.1469-8749.2008.03119.x

26. Redman TA, Gibson N, Finn JC, Bremner AP, Valentine J, Thickbroom GW. Upper limb corticomotor projections and physiological changes that occur with botulinum toxin-A therapy in children with hemiplegic cerebral palsy. Eur J Neurol (2008) 15:787-91. doi:10.1111/j.1468-1331.2008.02194.x

27. Staudt M, Ticini LF, Grodd W, Krägeloh-Mann I, Karnath H-O. Functional topography of early periventricular brain lesions in relation to cytoarchitectonic probabilistic maps. Brain Lang (2008) 106(3):177-83. doi:10.1016/j. bandl.2008.01.007

28. Vry J, Linder-Lucht M, Berweck S, Bonati U, Hodapp M, Uhl M, et al. Altered cortical inhibitory function in children with spastic diplegia: a TMS study. Exp Brain Res (2008) 186(4):611-8. doi:10.1007/s00221-007-1267-7

29. Pilato F, Dileone M, Capone F, Profice P, Caulo M, Battaglia D, et al. Unaffected motor cortex remodeling after hemispherectomy in an epileptic cerebral palsy patient. A TMS and fMRI study. Epilepsy Res (2009) 85(2-3):243-51. doi:10.1016/j.eplepsyres.2009.03.016

30. Walther M, Juenger H, Kuhnke N, Wilke M, Brodbeck V, Berweck S, et al. Motor cortex plasticity in ischemic perinatal stroke: a transcranial magnetic stimulation and functional MRI study. Pediatr Neurol (2009) 41(3):171-8. doi:10.1016/j.pediatrneurol.2009.04.006

31. Wilke M, Staudt M, Juenger H, Grodd W, Braun C, Krägeloh-Mann I. Somatosensory system in two types of motor reorganization in congenital hemiparesis: topography and function. Hum Brain Mapp (2009) 30(3):776-88. doi:10.1002/hbm.20545

32. Wittenberg GF. Motor mapping in cerebral palsy. Dev Med Child Neurol (2009) 51(Suppl 4):134-9. doi:10.1111/j.1469-8749.2009.03426.x

33. Holmström L, Vollmer B, Tedroff K, Islam M, Persson JK, Kits A, et al. Hand function in relation to brain lesions and corticomotor-projection pattern in children with unilateral cerebral palsy. Dev Med Child Neurol (2010) 52(2):145-52. doi:10.1111/j.1469-8749.2009.03496.x

34. Kesar TM, Sawaki L, Burdette JH, Cabrera MN, Kolaski K, Smith BP, et al. Motor cortical functional geometry in cerebral palsy and its relationship to disability. Clin Neurophysiol (2012) 123(7):1383-90. doi:10.1016/j.clinph. 2011.11.005

35. van der Aa NE, Verhage $\mathrm{CH}$, Groenendaal F, Vermeulen RJ, de Bode S, van Nieuwenhuizen $\mathrm{O}$, et al. Neonatal neuroimaging predicts recruitment of contralesional corticospinal tracts following perinatal brain injury. Dev Med Child Neurol (2013) 55(8):707-12. doi:10.1111/dmcn.12160

36. Yang JF, Livingstone D, Brunton K, Kim D, Lopetinsky B, Roy F, et al. Training to enhance walking in children with cerebral palsy: are we missing the window of opportunity? Semin Pediatr Neurol (2013) 20(2):106-15. doi:10.1016/j. spen.2013.06.011

37. Flamand VH, Schneider C. Noninvasive and painless magnetic stimulation of nerves improved brain motor function and mobility in a cerebral palsy case. Arch Phys Med Rehabil (2014) 95(10):1984-90. doi:10.1016/j.apmr.2014.05.014

38. Islam M, Nordstrand L, Holmström L, Kits A, Forssberg H, Eliasson A-C. Is outcome of constraint-induced movement therapy in unilateral cerebral palsy dependent on corticomotor projection pattern and brain lesion characteristics? Dev Med Child Neurol (2014) 56(3):252-8. doi:10.1111/dmcn.12353

39. Mackey A, Stinear C, Stott S, Byblow WD. Upper limb function and cortical organization in youth with unilateral cerebral palsy. Front Neurol (2014) 5:117. doi:10.3389/fneur.2014.00117

40. Pihko E, Nevalainen P, Vaalto S, Laaksonen K, Mäenpää H, Valanne L, et al. Reactivity of sensorimotor oscillations is altered in children with hemiplegic 
cerebral palsy: a magnetoencephalographic study. Hum Brain Mapp (2014) 35(8):4105-17. doi:10.1002/hbm.22462

41. Bleyenheuft Y, Dricot L, Gilis N, Kuo HC, Grandin C, Bleyenheuft C, et al. Capturing neuroplastic changes after bimanual intensive rehabilitation in children with unilateral spastic cerebral palsy: a combined DTI, TMS and fMRI pilot study. Res Dev Disabil (2015) 43-44:136-49. doi:10.1016/j.ridd.2015.06.014

42. Narayana S, Rezaie R, McAfee SS, Choudhri AF, Babajani-Feremi A, Fulton S, et al. Assessing motor function in young children with transcranial magnetic stimulation. Pediatr Neurol (2015) 52(1):94-103. doi:10.1016/j. pediatrneurol.2014.08.031

43. Baranello G, Rossi Sebastiano D, Pagliano E, et al. Hand function assessment in the first years of life in unilateral cerebral palsy: correlation with neuroimaging and cortico-spinal reorganization. Eur J Paediatr Neurol (2016) 20(1):114-24. doi:10.1016/j.ejpn.2015.09.005

44. Friel KM, Kuo H-C, Fuller J, Ferre CL, Brandão $M$, Carmel JB, et al. Skilled bimanual training drives motor cortex plasticity in children with unilateral cerebral palsy. Neurorehabil Neural Repair (2016) 30(9):834-44. doi:10.1177/1545968315625838

45. Kuo HC, Ferre CL, Carmel JB, Gowatsky JL, Stanford AD, Rowny SB, et al. Using diffusion tensor imaging to identify corticospinal tract projection patterns in children with unilateral spastic cerebral palsy. Dev Med Child Neurol (2017) 59(1):65-71. doi:10.1111/dmcn.13192

46. Valle AC, Dionisio K, Pitskel NB, Pascual-Leone A, Orsati F, Ferreira MJ, et al. Low and high frequency repetitive transcranial magnetic stimulation for the treatment of spasticity. Dev Med Child Neurol (2007) 49(7):534-8. doi:10.1111/j.1469-8749.2007.00534.x

47. Kirton A, Deveber G, Gunraj C, Chen R. Neurocardiogenic syncope complicating pediatric transcranial magnetic stimulation. Pediatr Neurol (2008) 39(3):196-7. doi:10.1016/j.pediatrneurol.2008.06.004

48. Kirton A, Chen R, Friefeld S, Gunraj C, Pontigon AM, deVeber G. Contralesional repetitive transcranial magnetic stimulation for chronic hemiparesis in subcortical paediatric stroke: a randomised trial. Lancet Neurol (2008) 7(6):507-13. doi:10.1016/S1474-4422(08)70096-6

49. Gillick BT, Krach LE, Feyma T, Rich TL, Moberg K, Thomas W, et al. Primed low-frequency repetitive transcranial magnetic stimulation and constraintinduced movement therapy in pediatric hemiparesis: a randomized controlled trial. Dev Med Child Neurol (2014) 56(1):44-52. doi:10.1111/dmcn.12243

50. Kirton A, Andersen J, Herrero M, Nettel-Aguirre A, Carsolio L, Damji O, et al. Brain stimulation and constraint for perinatal stroke hemiparesis: the PLASTIC CHAMPS trial. Neurology (2016) 86(18):1659-67. doi:10.1212/ WNL.0000000000002646

51. Guo Z, Xing G, He B, Chen H, Ou J, McClure MA, et al. Dynamic modulation of rTMS on functional connectivity and functional network connectivity to children with cerebral palsy: a case report. Neuroreport (2016) 27(4):284-8. doi:10.1097/WNR.0000000000000534

52. Gupta M, Lal Rajak B, Bhatia D, Mukherjee A. Effect of r-TMS over standard therapy in decreasing muscle tone of spastic cerebral palsy patients. J Med Eng Technol (2016) 40(4):210-6. doi:10.3109/03091902.2016.1161854

53. Young SJ, Bertucco M, Sheehan-stross R, Sanger TD. Cathodal transcranial direct current stimulation in children with dystonia: a pilot open-label trial. J Child Neurol (2013) 28(10):1238-44. doi:10.1177/0883073812460092

54. Aree-uea B, Auvichayapat N, Janyacharoen T, Siritaratiwat W, Amatachaya A, Prasertnoo J, et al. Reduction of spasticity in cerebral palsy by anodal transcranial direct current stimulation. J Med Assoc Thai (2014) 97(9):954-62.

55. Duarte NdAC, Grecco LAC, Galli M, Fregni F, Oliveira CS. Effect of transcranial direct-current stimulation combined with treadmill training on balance and functional performance in children with cerebral palsy: a double-blind randomized controlled trial. PLoS One (2014) 9(8):e105777. doi:10.1371/ journal.pone.0105777

56. Ferreira S, Rezende F, Dumont A, Ferreira L, Lazzari R, Oliveira C. Effect of a single session of transcranial direct current stimulation combined with virtual reality training on functional mobility in children with cerebral palsy: a randomized, controlled, double-blind, clinical trial. MTP Rehab J (2014) 23:1033-48.

57. Grecco LAC, Duarte NdAC, Mendonça ME, Cimolin V, Galli M, Fregni F, et al. Transcranial direct current stimulation during treadmill training in children with cerebral palsy: a randomized controlled double-blind clinical trial. Res Dev Disabil (2014) 35(11):2840-8. doi:10.1016/j.ridd. 2014.07.030
58. Grecco LAC, Duarte NAC, Zanon N, Galli M, Fregni F, Oliveira CS. Effect of a single session of transcranial direct-current stimulation on balance and spatiotemporal gait variables in children with cerebral palsy: a randomized sham-controlled study. Braz J Phys Ther (2014) 18(5):419-27. doi:10.1590/ bjpt-rbf.2014.0053

59. Grecco LAC, E Mendonça M, Duarte NAC, Zanon N, Fregni F, Oliveira CS. Transcranial direct current stimulation combined with treadmill gait training in delayed neuro-psychomotor development. J Phys Ther Sci (2014) 26(6): 945-50. doi:10.1589/jpts.26.945

60. Young SJ, Bertucco M, Sanger TD. Cathodal transcranial direct current stimulation in children with dystonia: a sham-controlled study. J Child Neurol (2014) 29(2):232-9. doi:10.1177/0883073813492385

61. Bhanpuri NH, Bertucco M, Young SJ, Lee AA, Sanger TD. Multiday transcranial direct current stimulation causes clinically insignificant changes in childhood dystonia: a pilot study. J Child Neurol (2015) 30(12):1604-15. doi:10.1177/0883073815575369

62. Ekici B. Transcranial direct current stimulation-induced seizure: analysis of a case. Clin EEG Neurosci (2015) 46(2):169. doi:10.1177/1550059414540647

63. Gillick BT, Feyma T, Menk J, Usset M, Vaith A, Wood TJ, et al. Safety and feasibility of transcranial direct current stimulation in pediatric hemiparesis: randomized controlled preliminary study. Phys Ther (2014) 95(3):337-49. doi:10.2522/ptj.20130565

64. Grecco LAC, de Almeida Carvalho Duarte N, Mendonça ME, Galli M, Fregni F, Oliveira CS. Effects of anodal transcranial direct current stimulation combined with virtual reality for improving gait in children with spastic diparetic cerebral palsy: a pilot, randomized, controlled, double-blind, clinical trial. Clin Rehabil (2015) 29(12):1212-23. doi:10.1177/0269215514566997

65. Lazzari RD, Politti F, Santos CA, Dumont AJ, Rezende FL, Grecco LA, et al. Effect of a single session of transcranial direct-current stimulation combined with virtual reality training on the balance of children with cerebral palsy: a randomized, controlled, double-blind trial. J Phys Ther Sci (2015) 27(3): 763-8. doi:10.1589/jpts.27.763

66. Carvalho Lima VLC, Collange Grecco LA, Marques VC, Fregni F, Brandão de Ávila CR. Transcranial direct current stimulation combined with integrative speech therapy in a child with cerebral palsy: a case report. J Bodyw Mov Ther (2016) 20(2):252-7. doi:10.1016/j.jbmt.2015.03.007

67. Grecco LAC, Oliveira CS, Duarte NdAC, Lima VLCC, Zanon N, Fregni F. Cerebellar transcranial direct current stimulation in children with ataxic cerebral palsy: a sham-controlled, crossover, pilot study. Dev Neurorehabil (2016) 8423:1-7. doi:10.3109/17518423.2016.1139639

68. Rossi S, Hallett M, Rossini PM, Pascual-Leone A; Safety of TMS Consensus Group. Safety, ethical considerations, and application guidelines for the use of transcranial magnetic stimulation in clinical practice and research. Clin Neurophysiol (2009) 120(12):2008-39. doi:10.1016/j.clinph.2009.08.016

69. Epstein C, Wasserman E, Ziemann U. The Oxford Handbook of Transcranial Magnetic Stimulation. New York: Oxford (2008).

70. Quintana $\mathrm{H}$. Transcranial magnetic stimulation in persons younger than the age of 18. J ECT (2005) 21(2):88-95. doi:10.1097/01.yct.0000162556.02720.58

71. Kirton A. Advancing non-invasive neuromodulation clinical trials in children: lessons from perinatal stroke. Eur J Paediatr Neurol (2017) 21(1):75-103. doi:10.1016/j.ejpn.2016.07.002

72. Palm U, Segmiller FM, Epple AN, Freisleder FJ, Koutsouleris N, SchulteKörne G, et al. Transcranial direct current stimulation in children and adolescents: a comprehensive review. J Neural Transm (2016) 123(10):1219-34. doi:10.1007/s00702-016-1572-z

73. Russo C, Souza Carneiro MI, Bolognini N, Fregni F. Safety review of transcranial direct current stimulation in stroke. Neuromodulation (2017) 20(3):215-22. doi:10.1111/ner.12574

74. Tekturk P, Erdogan ET, Kurt A, Kocagoncu E, Kucuk Z, Kinay D, et al. Transcranial direct current stimulation improves seizure control in patients with Rasmussen encephalitis. Epileptic Disord (2016) 18(1):58-66. doi:10.1684/ epd.2016.0796

75. Juenger H, Kuhnke N, Braun C, Ummenhofer F, Wilke M, Walther M, et al. Two types of exercise-induced neuroplasticity in congenital hemiparesis: a transcranial magnetic stimulation, functional MRI, and magnetoencephalography study. Dev Med Child Neurol (2013) 55(10):941-51. doi:10.1111/dmcn.12209

76. Kitchen L, Westmacott R, Friefeld S, MacGregor D, Curtis R, Allen A, et al. The pediatric stroke outcome measure: a validation and reliability study. Stroke (2012) 43(6):1602-8. doi:10.1161/STROKEAHA.111.639583 
77. Krumlinde-Sundholm L. Reporting outcomes of the Assisting Hand Assessment: what scale should be used? Dev Med Child Neurol (2012) 54(9):807-8. doi:10.1111/j.1469-8749.2012.04361.x

78. Weinberg W, Harper C, Emslie G. Weinberg Depression Scale for Children and Adolescents: Examiner's Manual. Austin, TX: Pro-Ed (1998).

79. Hoffmann TC, Glasziou PP, Boutron I, Milne R, Perera R, Moher D, et al. Better reporting of interventions: template for intervention description and replication (TIDieR) checklist and guide. BMJ (2014) 348:g1687. doi:10.1136/bmj.g1687

80. Bladin CF, Alexandrov AV, Bellavance A, Bornstein N, Chambers B, Coté R, et al. Seizures after stroke: a prospective multicenter study. Arch Neurol (2000) 57(11):1617-22. doi:10.1001/archneur.57.11.1617

81. Sellier E, Uldall P, Calado E, Sigurdardottir S, Torrioli MG, Platt MJ, et al. Epilepsy and cerebral palsy: characteristics and trends in children born in 1976-1998. Eur J Paediatr Neurol (2012) 16(1):48-55. doi:10.1016/j.ejpn. 2011.10.003

82. Ramos-Lizana J, Aguirre-Rodríguez J, Aguilera-López P, CassinelloGarcía E. Recurrence risk after withdrawal of antiepileptic drugs in children with epilepsy: a prospective study. Eur J Paediatr Neurol (2010) 14(2):116-24. doi:10.1016/j.ejpn.2009.05.006

83. Rich TL, Menk JS, Rudser KD, Chen M, Meekins GD, Peña E, et al. Determining electrode placement for transcranial direct current stimulation: a comparison of EEG- versus TMS-guided methods. Clin EEG Neurosci (2017) 48(6): 367-75. doi:10.1177/1550059417709177

84. Strozzi I, Nolan SJ, Sperling MR, Wingerchuk DM, Sirven J. Early versus late antiepileptic drug withdrawal for people with epilepsy in remission. Cochrane Database Syst Rev (2015) (2):CD001902. doi:10.1002/14651858. CD001902.pub2

85. Smorenburg AR, Gordon AM, Kuo HC, Ferre CL, Brandao M, Bleyenheuft Y, et al. Does corticospinal tract connectivity influence the response to intensive bimanual therapy in children with unilateral cerebral palsy? Neurorehabil Neural Repair (2017) 31(3):250-60. doi:10.1177/1545968316675427
86. Fox CK, Glass HC, Sidney S, Smith SE, Fullerton HJ. Neonatal seizures triple the risk of a remote seizure after perinatal ischemic stroke. Neurology (2016) 86(23):2179-86. doi:10.1212/WNL.0000000000002739

87. Tang A, Thickbroom G, Rodger J. Repetitive transcranial magnetic stimulation of the brain: mechanisms from animal and experimental models. Neuroscientist (2015) 23(1):82-94. doi:10.1177/1073858415618897

88. Zangen A, Roth Y, Voller B, Hallett M. Transcranial magnetic stimulation of deep brain regions: evidence for efficacy of the H-coil. Clin Neurophysiol (2005) 116(4):775-9. doi:10.1016/j.clinph.2004.11.008

89. Garvey MA, Kaczynski KJ, Becker DA, Bartko JJ. Subjective reactions of children to single-pulse transcranial magnetic stimulation. J Child Neurol (2001) 16(12):891-4. doi:10.1177/088307380101601205

90. Garvey MA, Gilbert DL. Transcranial magnetic stimulation in children. Eur J Paediatr Neurol (2004) 8(1):7-19. doi:10.1016/j.ejpn.2003.11.002

91. Rich TL, Menk J, Krach LE, Feyma T, Gillick BT. Repetitive transcranial magnetic stimulation/behavioral intervention clinical trial: long-term follow-up of outcomes in congenital hemiparesis. J Child Adolesc Psychopharmacol (2016) 26(7):598-605. doi:10.1089/cap.2015.0157

Conflict of Interest Statement: The authors declare that the research was conducted in the absence of any commercial or financial relationships that could be construed as a potential conflict of interest.

The reviewer RF and handling editor declared their shared affiliation.

Copyright (๑) 2018 Gillick, Gordon, Feyma, Krach, Carmel, Rich, Bleyenheuft and Friel. This is an open-access article distributed under the terms of the Creative Commons Attribution License (CC BY). The use, distribution or reproduction in other forums is permitted, provided the original author(s) and the copyright owner are credited and that the original publication in this journal is cited, in accordance with accepted academic practice. No use, distribution or reproduction is permitted which does not comply with these terms. 